\title{
Psychological investigation of spinal cord injury patients
}

\author{
Giorgio Scivoletto, Annelisa Petrelli, Lina Di Lucente and Vincenzo Castellano \\ IRCCS Ospedale di Riabilitazione S. Lucia, via Ardeatina 306, 00170 Roma-Italy
}

\begin{abstract}
Spinal cord injuries (SCI) often have psychological consequences, primarily anxiety and depression, which may interfere with rehabilitation possibilities, with adjustment to the impairment and therefore with the possibility of returning to previous familiar social life and work. To assess the degree of anxiety and depression in SCI patients, and to study the factors contributing to their genesis, 100 SCI in- and out-patients were administered questionnaires for self-rating anxiety and depression. A group of newly injured patients was followed up for a year after their discharge to study the evolution of the two psychological syndromes. These two psychological pathologies in SCI patients were respectively $13 \%$ (anxiety) and $16 \%$ (depression). Some characteristics were significantly associated with a higher risk of developing psychological distress: the presence of severe complications, the lack of autonomy, and low educational level. We did not observe any modification of the psychological picture over time. The lack of reduction in anxiety and depression over time could mean that the two pathologies are maintained by the obstacles SCI patients meet every day resulting from their neurological deficit. Our data justify the provision of psychological services for SCI patients in the rehabilitation centres; these services should still be available to the patients even after discharge.
\end{abstract}

Keywords: spinal cord injuries; anxiety; depression; psychological distress

\section{Introduction}

Spinal cord injuries (SCI) often have psychological consequences due to loss of functions, to alterations in the urinary tract and sexual functioning and to other symptoms such as pain, loss of social and work status and to a decrease in future expectations. ${ }^{1,2}$ The reported incidence of these pathologies varies from author to author: in the initial studies anxiety and most of all depression were considered almost inevitable consequences of $\mathrm{SCI}^{3}$, but today many authors report depression in only $20-45 \%$ of patients. ${ }^{4-6}$

The relevance of the psychological consequences is demonstrated by the rate of suicide and divorce among SCI patients; both are greater than in the general population and are on the increase. ${ }^{7}$ Anxiety and depression may have a considerable impact on functioning. Severe depressive episodes can cause health problems such as loss of weight, social problems such as loss of job and financial problems. Depressive episodes can be even more negative for those with functional losses (for example SCI patients). Tate et $a l^{8}$ demonstrated that depressed SCI patients spend more hours in bed, less time out of the house and need more medical assistance and nursing than non-depressed SCI patients.

Correspondence: Dott. G Scivoletto
The aim of this study was to investigate the psychological impact of spinal cord injury. We assessed the prevalence rates of SCI patients' anxiety and depression and examined some social, demographic and patient characteristics in order to determine their impact on psychological adjustment. ${ }^{9}$ We also followed a group of newly injured patients after their discharge from our hospital to see if there were differences in anxiety and depression over time and if our data were consistent with the traditional models of adjustment to SCI.

\section{Patients and methods}

\section{Patients}

One hundred patients with spinal cord injury of traumatic and non traumatic aetiology were assessed (79 males, 21 females, mean age 36 years) (Tables 1 and 2). Most of the patients (83\%) had lesions of the thoracic or lumbo-sacral spine $(33$ T1-T6 level, 35 T7-T12, 15 lumbo-sacral); 17 patients had a lesion of the cervical spine. With regard to the completeness of the lesion, 71 patients had an ASIA impairment scale A lesion ${ }^{10}, 13$ an ASIA B, 16 an ASIA D. At the time of assessment the distance from the lesion was between 3 months and 28 years (mean 4.6 years). None of our patients had a history of major psychiatric (major depression, schizophrenia) or cognitive disorders (head 
Table 1 Patients characteristics $(\mathrm{n}=100)$

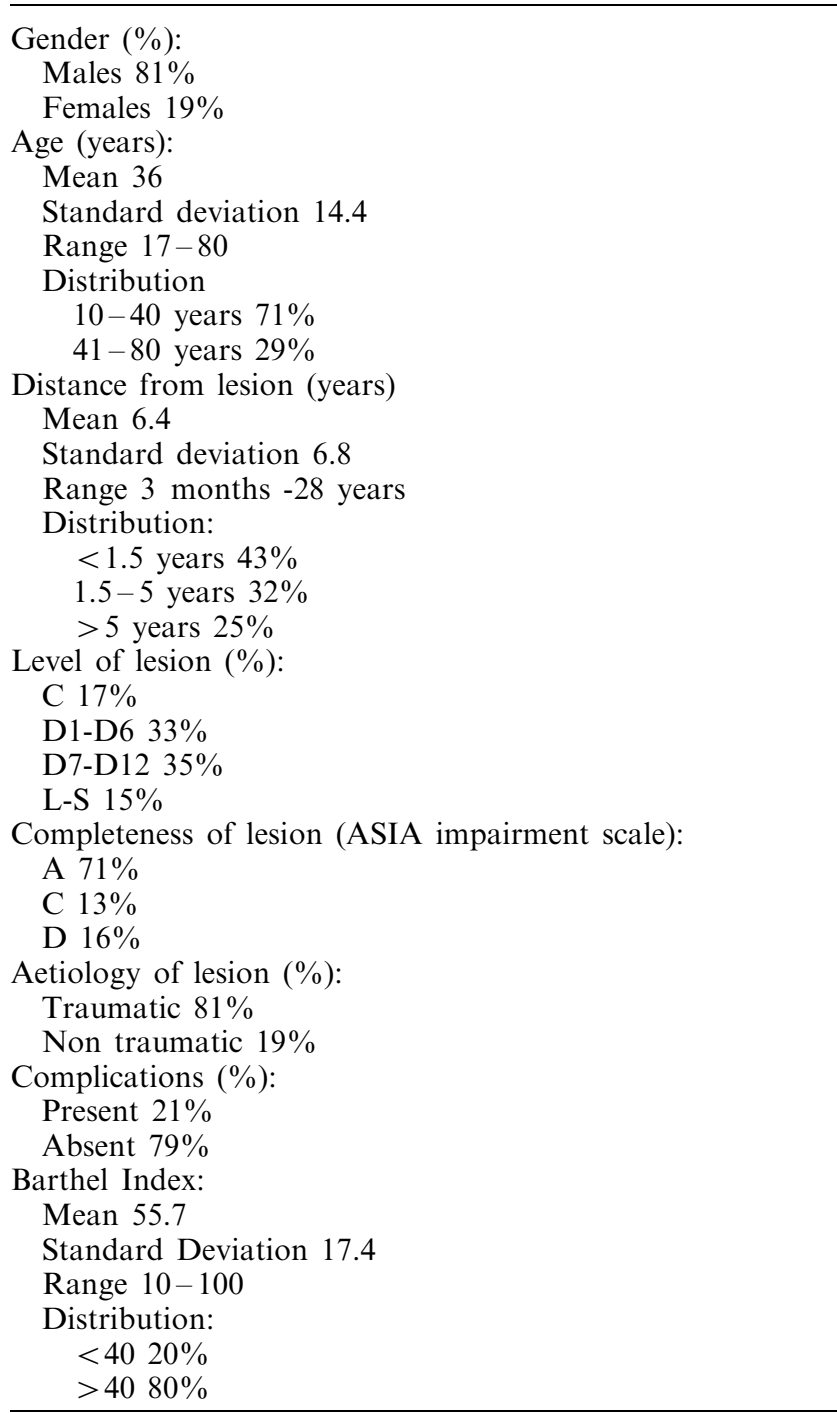

injury, mental retardation, organic mental disorders) that could affect the validity of their test responses.

A group of 27 newly injured ( $<6$ months) patients was followed up over time; the first evaluation was made as soon as the patients were mobile in a wheelchair.

\section{Methods}

All patients underwent:

- demographic and social inquiry;

- complete physical and neurological examination;

- the Spielberger State-Trait - Anxiety Inventory XI;

- the Depression Questionnaire (QD) of the Cognitive Behavioural Assessment. ${ }^{12}$

The STAI XI is a 20-item self-report measure of state anxiety; it has been widely used to study anxiety, also in SCI patients; ${ }^{1}$ it has a test-retest reliability of 0.68
Table 2 Social and demographic characteristics

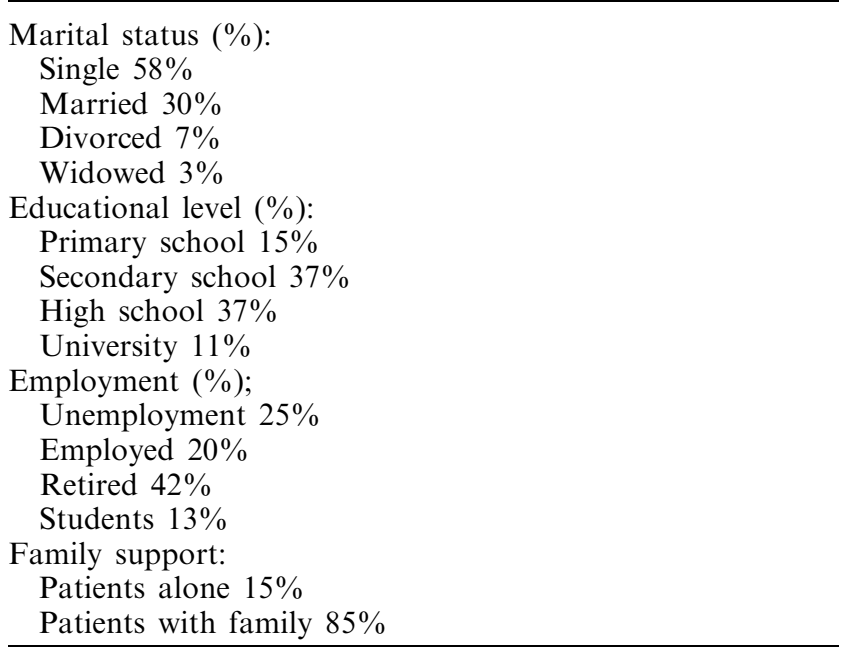

and 0.59 ( 7 and 30 days) ${ }^{12}$ the internal consistency (alpha coefficient) is $0.92 .{ }^{12}$ The QD questionnaire is a well validated self-report measure of depression consisting of 24 items adapted from the Beck Depression Inventory and the Zung Self-Rating Depression Scale; ${ }^{12,13}$ test-retest reliability is 0.88 and 0.72 ( 7 and 30 days) and the alpha coefficient $0.86 .^{12}$

\section{Statistical analysis}

We first evaluated the overall prevalence of anxiety and depression in the patients' group; the data of each patient were compared to the norms of the two tests (presented by the authors of the tests, ${ }^{12}$ ) the mean scores of a normal population of uninjured subjects comparable for age and sex. As already done in other studies, ${ }^{1}$ we choose as 'cut-off' score a score equivalent to one standard deviation above the mean of the norms. Patients were then defined anxious and depressed when their scores on the two scales were higher than the mean of uninjured subjects plus one standard deviation.

We then divided the SCI patients into groups on the basis of:

- age: $10-40$ years vs 41-80 years;

- sex: males vs females;

- distance from the lesion: <1.5 years vs $1.5-5$ years $v s>5$ years;

- the SCI level: C vs D1-6 vs D6-12 vs L-S;

- the completeness of the lesion: ASIA A vs C vs D;

- the presence or absence of severe complications such as pain, decubitus ulcers, paraosteoarthropathies, and repeated, severe urinary tract infections;

- level of autonomy, evaluated by means of the Barthel Index: ${ }^{14}<40$ vs $>40$;

- marital status: single vs married $v s$ divorced $v s$ widowed; 
- educational level: primary school vs secondary school vs high school vs university;

- occupation: students vs employed $v s$ unemployed vs retired;

- family support: patients with family $v s$ those alone;

- aetiology of the lesion: traumatic vs non traumatic.

The groups were compared with the chi square test to evaluate if there were differences in prevalence of anxiety and depression.

The group of newly injured patients was assessed on three occasions during the first year after injury and the results were evaluated by means of chi square test. An alpha rate of 0.05 or less was used.

\section{Results}

With regard to the overall prevalence of the two psychological syndromes 13 patients were anxious and 16 depressed. Tables 3 and 4 show the results of the various groups of patients (prevalence of anxiety and depression). With regard to medical characteristics, patients with severe complications were significantly more anxious and more depressed than the others $(P<0.005)$ and so were patients with a low level of autonomy $(P<0.05)$. With regard to social characteristics, patients with a low educational level, unemployed patients and those who lived alone had greater psychological distress than the others. No statistically significant difference was seen with regard to the other

Table 3 Prevalence of anxiety and depression in the various groups: medical variables

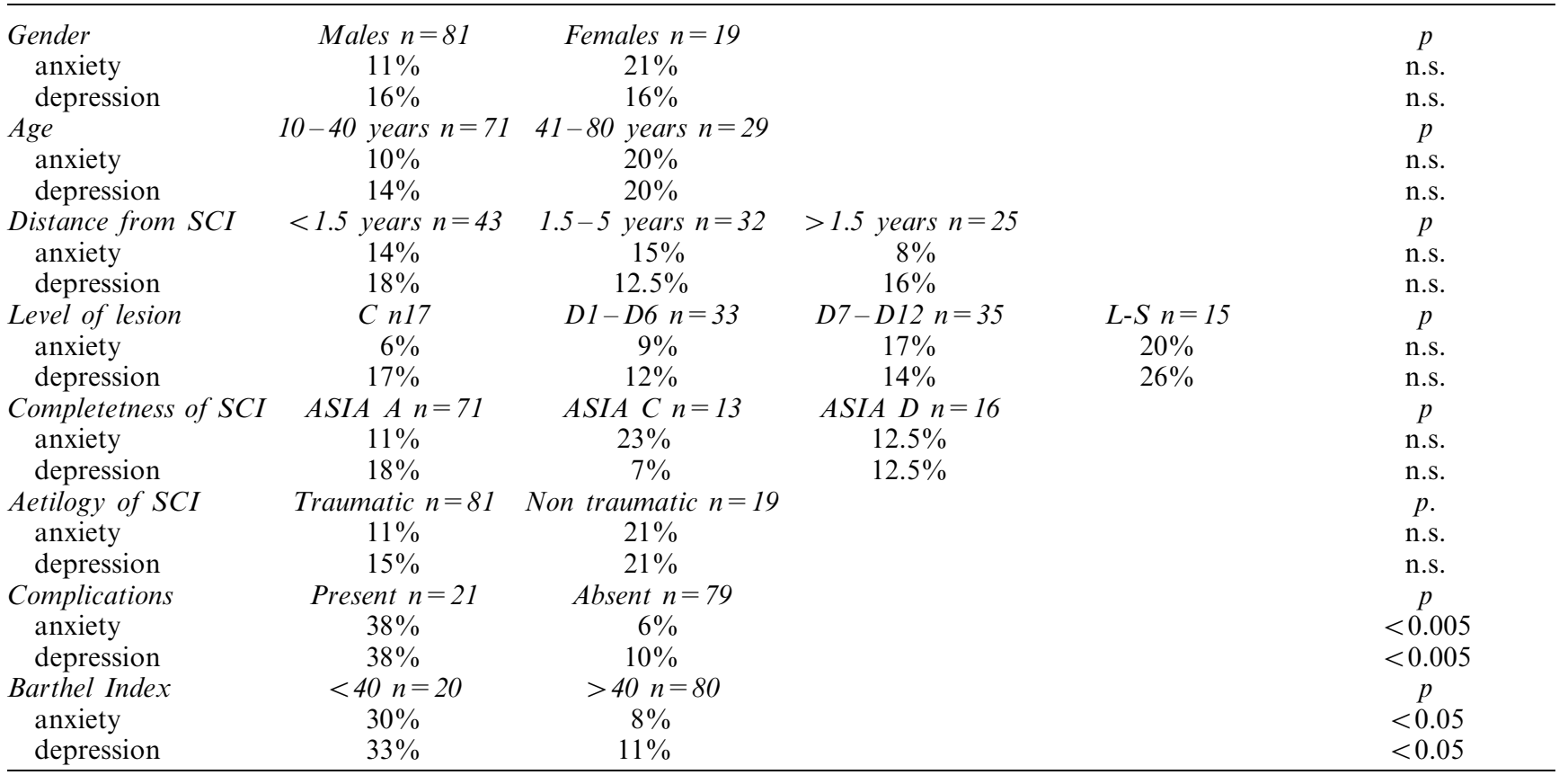

Table 4 Prevalence of anxiety and depression in the various groups: social and demographic variables

\begin{tabular}{|c|c|c|c|c|c|}
\hline Maritual status* & single $n=58$ & Married $n=30$ & Divorced $n=7$ & Widowed $n=3$ & $p$ \\
\hline anxiety & $7 \%$ & $20 \%$ & $28 \%$ & $33 \%$ & n.s. \\
\hline depression & $12 \%$ & $20 \%$ & $42 \%$ & $0 \%$ & n.s. \\
\hline Educational level & Primary school $n=15$ & Secondary school $n=37$ & High school $n=37$ & University $n=11$ & $p$ \\
\hline anxiety & $33 \%$ & $11 \%$ & $8 \%$ & $9 \%$ & $<0.05 * *$ \\
\hline depression & $40 \%$ & $13 \%$ & $13 \%$ & $0 \%$ & $<0.05 * * *$ \\
\hline Employment & Unemployed $n=25$ & Employed $n=20$ & Retired $n=42$ & Students $n=13$ & $p$ \\
\hline anxiety & $12 \%$ & $10 \%$ & $19 \%$ & 0 & n.s. \\
\hline depression & $28 \%$ & $15 \%$ & $14 \%$ & 0 & $<0.05 * * * *$ \\
\hline Family support & Patients alone $n=15$ & Patients with family $n=85$ & & & $p$ \\
\hline anxiety & $13 \%$ & $13 \%$ & & & n.s. \\
\hline depression & $33 \%$ & $13 \%$ & & & $<0.05$ \\
\hline
\end{tabular}

*statistical analysis was performed only between single and married patients, because of small number of patients in other groups. **between primary and high school. ***between primary school and the other three groups. ****between unemployed and students 
variables: age, sex, aetiology, level and completeness of the lesion, marital status.

Table 5 shows results of newly injured patients on the three assessments: no statistically significant differences were observed.

\section{Discussion}

Depending on the extent of their loss, SCI patients often present anxiety and depression. ${ }^{15-17}$ Apart from immobility and loss of sensation, SCI patients experience other unpleasant effects, such as pain and impaired urinary and sexual functioning; ${ }^{2}$ these physical symptoms often result in vocational and social losses.

Knowledge about adjustment to SCI is still incomplete; ${ }^{18}$ previous opinions have suggested that psychological distress, in particular depression, is a universal reaction following $\mathrm{SCI}{ }^{3}$ on the contrary current literature reports the presence of depression in only $20-40 \%$ of patients. ${ }^{4,5}$

A number of variables have been described in relation to anxiety and depression: age (older patients tend to be less well adjusted), ${ }^{19}$ education (low education is associated with worse adjustment), ${ }^{9}$ completeness and level of lesion, ${ }^{20}$ gender (male patients are more anxious and female patients more depressed) ${ }^{8,21}$ and marital status.

Our results are comparable to those of other studies even though the prevalence rates of anxiety and depression are lower than those reported elsewhere. None of our patients presented severe psychiatric disorders (according to DSM III) as in Judd's study ${ }^{16}$ in which patients fulfilled the diagnostic criteria for major depression.

We studied a number of variables to see if they were associated with anxiety and depression in our patients. Only few of these resulted important for the pathogenesis of psychological distress. The first one is the presence of severe complications, probably because these complications interrupt life routines and restrict social roles. Another medical variable is the patients level of autonomy: a low level of autonomy on the wheelchair and in daily living activity is associated with higher levels of anxiety and depression, regardless of the level and the completeness of the lesion. Once again this could be due to reduction of social life resulting from lack of autonomy.

Table 5 Evolution of the two psychological syndromes over time (group of newly injured patients)

\begin{tabular}{lccc}
\hline $\begin{array}{l}\text { Prevalence } \\
\text { rates }\end{array}$ & $\begin{array}{c}\text { First } \\
\text { evaluation }\end{array}$ & $\begin{array}{c}\text { Second } \\
\text { evaluation }\end{array}$ & $\begin{array}{c}\text { Third } \\
\text { evaluation }\end{array}$ \\
\hline Anxiety & $15 \%$ & $18 \%$ & $17 \%$ \\
Depression & $17 \%$ & $16 \%$ & $19 \%$ \\
\hline
\end{tabular}

Another variable positively associated with both anxiety and depression is educational level: patients with low education are more anxious and more depressed; in our opinion this could be due to the difficulties that these patients encounter when looking for an occupation, resulting in further vocational and social losses. Also important are family support and the possibility of returning to work after discharge from the hospital.

Thus the psychological effects of SCI seem to depend mainly on the degree of autonomy and on the possibility to have a lifestyle as normal as possible, independently from all the other medical and social factors.

We believe that the more interesting data concerns the lack of significant changes in prevalence rates of anxiety and depression over time. As already observed by Craig, ${ }^{22}$ this lack is inconsistent with the usual models of adjustment, that is the stage models, which suggest that psychological distress should decrease over time as happens after mourning. A common example of the stages includes denial (in the first six months after the lesion), depression, anger and acceptance $^{23}$ and implies that the lesion itself is the primary factor influencing the adjustment. ${ }^{24}$

On the contrary, our data seem to demonstrate that psychological distress in SCI patients is caused and maintained by the difficulties the patients meet every day; daily living with a handicap such as that resulting from a SCI means the need to cope with architectural barriers, economic costs, vocational limitations, alterations in familiar and social role. These obstacles replace the difficulties met during hospitalisation (lack of privacy, separation from parents and relatives, dependence on nursing staff) and maintain anxiety and depression over time. ${ }^{25}$ In fact in our patients the variables associated with psychological distress were mostly those influencing the social and vocational life (presence of severe complications, level of autonomy, education, employment, family support), while the other medical variables appeared to be less important.

Psychological adjustment to SCI is a complex and extremely individual process, because of the interaction of organic variables (medical aspects), person variables (personality, capactity to manage stress) and environmental variables (family support and socioeconomic status). Thus, saying that time will lower depression and anxiety may be merely a simplification.

\section{Conclusions}

Our results have important implications for the treatment of SCI patients: first, they show that psychological distress is not an inevitable consequence of SCI, but is limited to $20 \%$ of patients; second, although not inevitable, anxiety and depression are maintained over time and do not decrease as previous models have suggested. In our opinion these data justify the provision of a psychological service in every hospital where SCI patients are treated. These services 
should be available to patients not only during hospitalisation, but also after their discharge.

\section{References}

1 Hancock KM, et al. Anxiety and depression over the first year of spinal cord injury: a longitudinal study. Paraplegia 1993; 31: $349-357$.

2 Engel GL. Psychological development in health and disease. Philadelphia: WB Saunders Company, 1962; pp 288-305.

3 Siller J. Psychological situation of the disabled with spinal cord injuries. Rehab Lit 1969; 30: 290-296.

4 Fullerton DT, Harvey RF, Klein MH, Howell TH. Psychiatric disorders in patients with spinal cord injuries. Arch Gen Psychiatry 1981; 38: $1361-1371$

5 Judd FK et al. Depression following spinal cord injury: A prospective in-patient study. Br J Psychiatry 1989; 154: 668-671.

6 Fuhrer MJ et al. Depressive symptomatology in persons with spinal cord injury who reside in the community. Arch Phys Med Rehabil 1993; 74: $255-260$.

7 Geisler WO, Jousse AT, Wynne-Jones M, Breithaupt SD. Survival in traumatic spinal cord injury. Paraplegia 1983; 21: $304-373$.

8 Tate D, Forcheimer M, Maynard F, Dujkers M. Predicting depression and psychological distress in persons with spinal cord injury based on indicator of handicap. Am J Phys Med Rehabil 1994; 73: $175-183$

9 Woodrich F, Patterson JB. Variables related to acceptance of disability in persons with spinal cord injuries. J Rehabil 1983; 49: $26-30$.

10 Di Tunno JF, Young W, Donovan WH, Creasey G. The international standards booklet for neurological and functional classification of spinal cord injury. Paraplegia 1994; 32: $70-80$.

11 Spielberger et al. Manual for the State-Trait Anxiety inventory. Palo Alto: Consulting Psychologist Press, 1983.

12 Sanavio E et al. CBA - 2.0 Scale Primarie. Manuale. Firenze: Organizzazioni Speciali, 1986.
13 Zotti AM, Bertolotti G, Meriggi A. L'assessment psicofisiologico ed il suo impiego nell'approccio riabilitativo. IV Congresso Nazionale di Biofeedback e Medicina Comportamentale (Atti), 1984.

14 Mahoney F, Barthel DW. Functional evaluation: the Barthel Index. Mar State Med J Rehab 1965, 2: 61-65.

15 Judd FK, Burrows GD. Liaison psychiatry in a spinal injuries unit. Paraplegia 1986a; 24: 6-19.

16 Judd FK, Burrows GD, Brown DJ. Depression following acute spinal cord injury. Paraplegia 1986b; 24: 358-363.

17 Somasundaram O, Balakrishnan S, Ravindran OS, Shanmugasundaram TK. A psychological study of spinal cord injured patients involved in the Madras Paraplegia Project. Paraplegia 1992; 30: $799-802$.

18 Stensman R. Adjustment to traumatic spinal cord injury. A longitudinal study of self-reported quality of life. Paraplegia 1994; 32: $416-422$

19 Kerr W, Thompson M. Acceptance of disability of sudden onset in paraplegia. Int J Paraplegia 1972; 10: $94-102$.

20 MacDonald MR, Nielson WR, Cameron MGP Depression and activity patterns of spinal cord injured persons living in the community. Arch Phys Med Rehabil 1987; 68: 339-343.

21 Rasdloff LS. The CES-D scale: a self-report depression scale for research in the general population. Appl Psychol Meas 1977; 1: $385-401$.

22 Craig AR, Hancock KM, Dickson HG. A longitudinal investigation into anxiety and depression in the first 2 years following a spinal cord injury. Paraplegia 1994; 32: 675-679.

23 Homan G. Psychological aspects of treatment and rehabilitation of the spinal injured person. Clin Orthop 1975; 112: $81-88$.

24 Frank RG, Elliot TR, Corcoran JR, Wonferlich SA. Depression after spinal cord injury: is it necessary? Clin Psychol Rev 1987; 7: $611-630$.

25 Trieschmann RB. Spinal cord injuries: psychological, social and vocational rehabilitation, 2nd Edition, Arizona: Scottsdale, 1988. 